\title{
Interactive Robots in Experimental Biology
}

3

4

5

6

7

8

9

10

11

12
Jens Krause ${ }^{1,2}$, Alan F.T. Winfield ${ }^{3} \&$ Jean-Louis Deneubourg $^{4}$

${ }^{1}$ Leibniz-Institute of Freshwater Ecology and Inland Fisheries, Department of Biology and Ecology of Fishes, 12587 Berlin, Germany;

${ }^{2}$ Humboldt-University of Berlin, Department for Crop and Animal Sciences, Philippstrasse 13, 10115 Berlin, Germany;

${ }^{3}$ Bristol Robotics Laboratory, University of the West of England, Coldharbour Lane, Bristol BS16 1QY, UK;

${ }^{4}$ Unit of Social Ecology, Campus Plaine - CP 231, Université libre de Bruxelles, Bd du Triomphe, B-1050 Brussels - Belgium

0

(1)

8 Corresponding author: Krause, J. (j.krause@igb-berlin.de), Leibniz Institute of Freshwater 9 Ecology and Inland Fisheries, Department of the Biology and Ecology of Fishes, Müggelseedamm 310, 12587 Berlin, Germany. 2 3 
Interactive robots have the potential to revolutionise the study of social behaviour because they provide a number of methodological advances. In interactions with live animals the behaviour of robots can be standardised, morphology and behaviour can be decoupled (so that different morphologies and behavioural strategies can be combined), behaviour can be manipulated in complex interaction sequences and models of behaviour can be embodied by the robot and thereby be tested. Furthermore, robots can be used as demonstrators in experiments on social learning. The opportunities that robots create for new experimental approaches have far-reaching consequences for research in fields such as mate choice, cooperation, social learning, personality studies and collective behaviour.

\section{Introduction to interactive robots}

Tinbergen [1] demonstrated that in some species of fish, birds and butterflies only simple stimuli were required to elicit territorial or mating behaviour that is normally only shown in response to male and female conspecifics. Insights into the mechanisms of social recognition coupled with technological advances suggest that robots can be developed for use in behavioural research to simulate con- and heterospecific behaviour. For the purposes of this review, we define a robot as a machine that is able to physically interact with its environment and perform some sequence of behaviours, either autonomously or by remote control. In recent years we have witnessed the transition from robots which, once set in motion, "blindly" follow a particular programme to ones that can interact with their environment, learn and even adapt [2-5]. This creates many opportunities for the use of robots in experimental biology, particularly when investigating social behaviour. One of the main challenges when investigating social behaviour is that the behaviour of individuals is dependent on that of their interaction partners. It is possible to infer certain rules or strategies from behavioural observations but unless we can manipulate the behaviour of individuals, this approach remains largely descriptive. One way to manipulate behaviour is to create robots that are accepted as con- or heterospecific and which can be programmed to carry out specific behavioural patterns. A related approach which serves the same purpose (of getting control over the behaviour of one or more individuals in a group or population) is to fit a live animal with interactive technology so that one animal in a group is effectively controlled as the 'robot' that interacts with its conspecifics. A brief overview of robots and interactive 
technologies is provided in Table 1.

Here, we give an overview of interactive robotics for use in experimental biology focusing on social behaviour. This approach has been successfully used across the animal kingdom ranging from studies on social insects [6,7] and cockroaches [4] to fish [8], birds [9] and mammals including humans [10-13]. Previous reviews on robots in biological research [1417] were less focussed on the interactive component which is a recent technological development and has become an important component of studies on collective behaviour [4,18-21]. We will identify important novel biological research questions that can be answered with the help of interactive robots and outline new directions for future developments in machine-animal interactions.

\section{Interactive technologies}

\section{$\underline{\text { Robots and computer animations }}$}

Robots are not the only way to create interactions with live animals. Animations in which virtual animals on a computer screen display realistic behaviours and interact with live animals have become an important tool for investigating animal behaviour [22-24]. This approach has provided many new insights, particularly in the areas of sexual selection and prey recognition [23]. Some of the major advantages of using virtual animals (compared to using real ones) are that it becomes possible to standardise the behaviour of display individuals in choice experiments and to de-couple behaviour and morphology (to present visual stimuli in isolation or combination). For example, this approach made it possible to identify the role of male ornaments in mate choice of female swordtail fish (Xiphophorus helleri) [25]. The use of animation allowed the presentation of the same behaviour by males versus ones without a sword-tail but with an enlarged body to compensate for loss of surface area. This decoupled morphology and behaviour and demonstrated that the sword does not simply help to increase perception of male size in females.

However, animations with virtual animals also have many limitations because they are largely restricted to the use of visual stimuli in two dimensions. Many animal interactions require other or additional sensory input (for example, fish species can usually sense the presence of conspecifics through the lateral line (via mechanical stimuli) and most species of social insects require olfactory stimuli for social recognition) and take place in three dimensions. They require the physical presence of a con- or heterospecific to fight, mate or 
cooperate with and these types of interaction by their very nature cannot be established with a virtual partner and require a robot.

Robots can provide solutions to some of the issues connected with virtual animals but also have some potential problems of their own. Developing robots that are accepted as conspecifics may not be equally straightforward in different species (depending on which sensory channels are used for social recognition, the size of the species and its cognitive abilities to name but a few factors) and the difficulty of implementing movements and responses varies considerably. Building robots can also be time-consuming and in some cases expensive and often requires collaboration with scientists in other disciplines. Despite these potential problems there are in principle no limits to how realistic we can make a robot appear like a con-or heterospecific in terms of its behaviour and morphology.

\section{$\underline{\text { Smart collars and cyborgs }}$}

New devices such as electronic collars make it possible to get control over some aspects of the behaviour of animals and therefore allow behavioural manipulations without investing substantial effort to create an animation or build a robot. These devices were originally developed for domestic animals which can be fitted with a "smart" collar that produces an adverse stimulus (sound, odour, mild electric shock) if the animal comes too close to the boundary of its designated area where a wire has been buried that communicates with the collar. This technology is already commercially available for domestic dogs. However, for larger scale use in cattle herding the collar usually contains a GPS unit that can determine the location of the animal which is more flexible and cost-effective. An example of such work is the virtual fence project which promotes the spatial control of livestock by means of smart collars instead of fences [26-28]. Additionally, by making use of social hierarchies and collective behaviour, only a small fraction of the total herd usually needs to be equipped [18, 29-30].

However, while it is possible to exert some influence on the behaviour of animals in this way (i.e. they can be maintained in a certain area) it does not produce the kind of fine-control that a robot provides. The strength of the response of the animal and its movement details cannot be reliably controlled with a collar and are left to chance. This means that if the same individual is given the same collar stimulus repeatedly, it may still produce a variable behavioural output. In addition, there is often considerable inter-individual variation in response to the collar stimuli [27]. An alternative to smart collars is the work on "cyborgs" ([31-33], Box 1). 
Making robots interactive

There are a number of common, basic requirements that must be fulfilled if interaction with live animals is to be possible. The behaviour of live animals needs to be monitored (e.g. through direct observation or an automated camera system) to provide the sensory basis for a response by the agent (virtual animal or robot). This sensory information is used to make a decision (usually made by a human observer or a computer) over how the agent should respond in the next time step. Depending on what the live animal does next this can potentially lead to a chain of interactions between animal and agent. Some researchers use a simple remote-control system to initiate a response in the robot when they want to create an interaction between live animal and robot [9]. This means the first two steps regarding sensory input and decision-making (discussed above) are operated by a human observer. This approach has the disadvantage that much is left to the judgement of the scientist operating the robot. More sophisticated systems give the robot sensory input, a control system and behavioural output so that it can make its own (standardised) decisions as to when and how to interact [4]. This approach can result in an autonomous robot where the animal and the robot interact without intervention from an observer [4]. As an alternative, the control system can be externalised in order to allow the experimenter to change the course of an interaction between robot and animal at any point (Box 2). For example, the experimenter could load a new interaction sequence if the context required it.

Furthermore, for the analysis of robot-animal interactions and the operation of remotecontrolled robots, $2 \mathrm{~d}$ or $3 \mathrm{~d}$ tracking of robot and animal(s) is vital and usually done via digital video cameras which are connected to a computer. While pattern recognition and tracking

160 have made great advances in recent years [34], fully automated tracking of multiple objects

161 (robots and/or animals) can still be surprisingly problematic under experimental conditions.

\section{Robots in behavioural experiments}

Interactive robots have the potential to revolutionise the way in which we perform experimental work with animals because they provide a number of important methodological advances. 


\section{$\underline{\text { Manipulation of interaction sequences }}$}

171 Interactive robots allow us to investigate entire interaction sequences where formerly scientists could only provide an animal with a single stimulus and then wait for a response.

173 Many if not most animal interactions involve behavioural sequences which were previously 174 difficult to test experimentally in a standardised way. Particularly relevant behavioural contexts that can involve lengthy interaction sequences include cooperation, courtship and agonistic behaviours and the fast-growing research area of collective behaviour.

Communal roosting is a wide-spread behaviour but little is known about how individuals agree on a location. To investigate the mechanisms of communal shelter-seeking in cockroaches (Periplaneta americana), robots were created that behaved like cockroaches and that were accepted as conspecifics (based on their odour) by the cockroaches ([4]; Fig. 1). The robots were autonomous and capable of recognising the shelters and the walls of the arena and of interacting with the cockroaches. The cockroaches prefer the darker of two shelters but in the presence of cockroach robots that 'preferred' the lighter shelter, they could be made to accept the lighter one more often than they normally would. The robots, despite their preference for the lighter shelter, occasionally followed the cockroaches and occupied the darker one. The experiments showed that the eventual outcome (adoption of the dark or light shelter) was a result of a complex interaction between robots and cockroaches. The non-linear nature of the decision-making process could result in either the cockroaches or the robots taking charge in the shelter selection process. Selecting a common shelter (from two alternatives) involved many interactions between cockroaches and robots over an extended time period.

Another promising area in which interaction sequences are particularly important is that of mating displays where a mixture of different signals are employed and where the actions of

194 the sexes are highly interdependent. Interactive robots could provide opportunities for 195 simulating different male courtship behaviours to evaluate their effect on females and 196 likewise different female responses to male courtship [35]. An example is the elegant work by 197 Patricelli et al. [9,36] in which robotic female bowerbirds (Ptilonorhynchus violaceus) were 198 used to investigate male courtship behaviour. A startle response in females significantly reduced the courtship intensity in males [35]. Patricelli et al. used a technique by which the researcher triggered the response of the robotic female by remote-control from a hide when the bowerbird male began courtship. Therefore the timing of the response was determined by the experimenter and depends on his/her accuracy of judgment. Given that the experimenter's 
perspective (from a hide) is likely to be different from that of the female robot which has a more direct and localised view, it would be an interesting challenge to provide the robot with local sensors that allow it to trigger its own startle behaviour in response to details of the male courtship display, may not even be perceptible by a human observer from a nearby hide.

\section{$\underline{\text { Using robots as leaders }}$}

Robots can be used to explore how animals select leaders and in which contexts they are willing to follow. In a study on decision-making behaviour remote-controlled fish models (and later a robotic fish) were used to demonstrate that the decision of which path to choose in a y-maze was based on a quorum [37]. If the robotic fish took the risky path (passing a predator model) and not the safe one, it was followed by a single fish but less often by groups of 2, 4 and 8 fish. To guide groups past the predator model, two (or more) robotic fish were required. Three robots generated no additional following (compared to two robots) supporting the idea that a quorum was already reached with two leaders. If the fish had to choose between two robotic fish that were different in appearance and which moved in different directions, the decision in favour of the more popular one dramatically increased as a function

\section{Robots for testing models of behaviour}

In the case of collective behaviours of fish schools and bird flocks there is no shortage in the literature of mechanistic models of these systems but a real lack of empirical data and experimental tests [39]. Interactive robots should be used here to critically assess these models and the assumptions they are based on. For example, in the debate on modelling collective behaviour some authors proposed metric interactions (i.e. individuals respond to the movements of near neighbours within a certain distance [40]) others proposed topological ones (i.e. individuals respond to fixed number of near neighbours largely regardless of distance [20]). To discriminate between the two model predictions a robotic fish was used that performed a sudden change in direction relative to that of the rest of the shoal. From the response of the shoal members it became clear that a topological model is more realistic [8]. This type of research required a robot that could enter a group and physically interact with its members.

\section{Conclusions and future perspectives}




\section{$238 \quad$ Selection of interactive technology}

239 Interactive technology offers a whole new range of possibilities for experimental work in 240 animal behaviour. Depending on the species, the research question and the budget, different 241 options for interactive robots are available starting from lab-based systems that allow the use 242 of different robots within relatively small spaces (Box 2) to fully autonomous devices (Fig. 1). 243 The approach used in creating "cyborg" insects (Box 1) is bound to become even more 244 sophisticated in the near future and should hold interesting possibilities for experimentalists 245 that require behavioural control over one or several individuals. The strength of the cyborg approach is that the animal itself is being used rather than a machine that resembles an animal. Interactions in social insects could be manipulated in this way to explore open questions in collective behaviour research [41-43]. For example, several projects used robotic honey bees to investigate the waggle dance and the onset of information cascades [44,7]. However, if fine-control of a worker becomes possible through the cyborg approach this could potentially open up new ways to further investigate this complex behaviour.

Electronic collar technology could be used to address a number of interesting questions and practical conservation issues. We can test predictions from the literature [18] as to what proportion in a group needs to be controlled to manipulate the whole group. In animals that have social hierarchies we could experimentally explore which individuals exert the greatest influence during movement decisions [45]. The applications of this technology in terms of

257 farm animals and domestic animals are clear and in some cases already widely explored.

258 However, there are two key areas where smart collar technology might be useful also for wildlife management: keeping large herbivores away from valuable crops and predators away from livestock. For example, one of the first free-ranging herds of European bison in Germany is supposed to be restricted to a particular woodland area in this way (Witte pers. commun.).

\section{Manipulation of interaction sequences}

265 We described a number of examples above in which interactive robots have been successfully used to investigate animal behaviour. Particularly in the contexts of cooperation and agonistic behaviour the use of interactive robots could pave the way for further progress. For example, in the case of predator inspection behaviour the place of one individual could be taken by an interactive robot which could follow different types of interaction programmes depending on which aspect of cooperation or defection should be simulated (e.g. risk-sharing by sharing the 
lead or return to the group). Box 2 shows that a methodology for this type of experiment exists [8]. By giving the robot different identities (through different body patterns or odours) it would also be possible to test whether individuals that frequently defect (while controlling for other behavioural or morphological differences) are avoided as partners for predator inspection in future. Furthermore, this approach could establish how many different cooperation partners can actually be remembered and for how long.

Agonistic behaviour in the form of territorial displays of individuals is another case in point. The behaviour of the rival males often strongly depends on what the opponent does [46-47] and this could potentially be investigated systematically with an interactive robot. For the study of winner and loser effects it might be possible to stage fights between robots that mimic conspecific males and to study what the audience (i.e. males or females that watch the behaviour) can learn from such interactions. The use of two robots for fight sequences would allow standardisation of interactions within and between fights so that we can control what each individual audience member watches at any time.

\section{$\underline{\text { Robots to embody personality types }}$}

Robots could be used to experimentally decouple behaviour and morphology by systematically manipulating different aspects of morphological and behavioural traits to investigate their relative importance. The latter could include personality type which would allow an assessment of the role of personalities in decision-making processes and in social networks [48]. Social networks can be generated on the basis of interactions, spatial proximity, relatedness or other factors [49]. Social network analysis provides us with many new metrics to characterize the social fine-structure of populations [49-50] and therefore with an opportunity to gain an understanding of the role that different personalities play in groups and populations regarding the transmission of information or disease or in terms of cooperation and policing of social conflicts [51,52]. How an individual can build up a certain network position and what influence this position offers could be experimentally tested through interactive robots providing novel insights into the social organisation of animals.

Different studies described the development of behavioural differentiation in groups (e.g. in cases where food accessibility was made difficult). For example, a proportion of individuals may specialize in stealing food from others, or in joining others that have already located food $[53,54]$. Introducing specialized robots that mimic producer-scrounger behaviour within the group might show how the proportion of different specialists is modified. Similarly in insect societies, the introduction of robots as workers and how these modify the pattern of 
division of labour could be investigated.

\section{$\underline{\text { Robots as demonstrators }}$}

308 The cross-disciplinary study of imitation and social learning in robots, humans and animals 309 has emerged in recent years [55]. Animal behaviour experiments would benefit enormously 310 from having robotic "demonstrators" to explore the transmission process of copying 311 behaviour. The experiments on leadership in fish decision-making discussed earlier are just 312 the beginning of this new field [37,38]. We described experiments on fish (in the section 313 Using robots as leaders) in which the phenotypic characteristics of leaders were manipulated 314 to explore the willingness of conspecifics to follow but this approach could be pushed further 315 to investigate also the willingness to copy behaviours and socially learn. Furthermore, the 316 manipulation of the demonstrator's behaviour could provide new important insights into what 317 information observers can extract from watching demonstrators (for example when exploiting 318 a food patch). Female robots could be a useful tool in experiments on mate choice copying. 319 The robot could simulate a preference for a particular male and the strength of this preference could be precisely controlled in a robot so that copying behaviour from females could be studied in detail. Robotic demonstrators could demonstrate behaviours with different error rates which would address the question of whether it is easier to learn from individuals that make mistakes.

324 Young animals can be imprinted on robots interacting with them [56]. An interesting area for application is the use of robots for guiding young of the year that have been imprinted on the robot (which embodies a parent) along a suitable migration route or away from danger. In the past geese, cranes and other species [57] have been imprinted on costumed humans (who mimic the parents species) and were trained to follow a light aircraft. This approach could potentially be expanded to other species and contexts with robots that mimic the respective species and can replace both humans and light aircraft.

\section{$\underline{\text { Swarm intelligence and swarm robotics }}$}

In the context of collective behaviour, swarm intelligence has attracted much interest [58, 59]. The role of the cognitive abilities of individuals in the decision-making process of groups is still relatively little understood which opens up many possibilities for experimental work.

336 How the information that individuals provide is processed could be investigated with robots that inject pre-selected bits of information into the decision-making process. This is not to say that this type of work can only be carried out with interactive robots. Several studies $[29,60]$ 
showed how trained or instructed individuals can be used to initiate new behaviours in groups. However, the latter does not provide the same degree of control as robots because of inter-individual and within-individual variation (e.g. due to changes in motivation).

Swarm robotics [61] is a rapidly expanding field of research which offers a number of interesting approaches to the study of animal behaviour. Automated recognition of social behaviours can be used to assess the behavioural repertoire of an individual or a species (similar to classical ethograms) and to calculate transition probabilities between different behaviours to develop dynamic models of the behavioural architecture of organisms [3]. Robots can then be used to embody these models. And going one step further, swarm robotics can facilitate the study of evolutionary processes as well by mutating and evolving robot social behaviour which can provide novel predictions for the study of communication and adaptive behaviour $[5,62,63]$. Symbrion is a project that goes even further by aiming to model, in a self-assembling swarm of robots, generic processes within biology such as morphogenesis, energy homeostasis, and immune responses to faults [64].

Interactive robots offer exciting new opportunities for experimental research. With the help of robots complex interaction sequences can be manipulated and behaviour and morphology can be decoupled. Robots can act as leaders and demonstrators and can potentially even be used to embody personality types in social networks. These methodological advances facilitate novel experimental work that will push the boundaries of behavioural research.

\section{Acknowledgements}

361 We thank Iain Couzin, Laurent Keller, Kevin Laland, Naomi Leonard, Chris Melhuish, Tommaso Pizzari, Guy Theraulaz, Ashley Ward, Barbara Webb, and four anonymous referees for their input. Funding was provided to JK by the NERC (NE/D011035/1).

\section{References}


3733 Balch, T. et al. (2006) How multi-robot systems research will accelerate our understanding 374 of social animal behavior. P. IEEE 94, 1445-11463

375

3764 Halloy, J. et al. (2007) Social integration of robots into groups of cockroaches to control 377 self-organised choices. Science 318, 55-1158

3795 Mitri, S. et al. (2009) The evolution of information suppression in communicating robots 380 with conflicting interests. Proc. Natl. Acad. Sci. U. S. A 106, 15786-15790

6 Michelsen, et al. (1992) How honeybees perceive communication dances, studied by means of a mechanical model. Behav. Ecol. Sociobiol. 30, 143-150

7 Landgraf, T. et al. (2008) Design and development of a robotic bee for the analysis of 386 honeybee dance communication. Appl. Bion. Biom. 5, 157-164

8 Faria, J.J. et al. (2010) A novel method for investigating the collective behaviour of fish: introducing "Robofish". Behav. Ecol. Sociobiol. 64, 1211-1218

9 Patricelli, G.L. et al. (2002) Male displays adjusted to female's response - Macho courtship

10 Vaughan, R. et al. (2000) Experiments in automatic flock control. Robo. Auton. Syst. 31, 109-117

11 Ishii, H. et al. (2006) Experimental study on task teaching to real rats through interaction 398 with a robotic rat. Lect. Not. Comp. Sci. 4095, 643-654

40012 Walters, M.L. et al. (2008) Avoiding the uncanny valley: robot appearance, personality 401 and consistency of behavior in an attention-seeking home scenario for a robot companion. 402 Auton. Robot. 24, 159-178 
40413 Dautenhahn, K. et al. (2009) KASPAR - A Minimally Expressive Humanoid Robot for 405 Human-Robot Interaction Research. Special Issue on "Humanoid Robots", App. Bion. Biom. 406 6: 369-397

407

14 Webb, B. (2000) What does robotics offer animal behaviour? Anim. Behav. 60, 545-558

409

15 Holland, O. and McFarland, D. (2001) Artificial Ethology. Oxford University Press

16 Knight, J. (2005) Animal behaviour: When robots go wild. Nature 434, 954-955

413

17 Webb, B. (2008) Using robots to understand animal behavior. Adv. Stud. Behav. 38, 1-58

18 Couzin, I.D. et al. (2005) Effective leadership and decision-making in animal groups on

417 the move. Nature 433, 513-516

19 Buhl, J. et al. (2006) From disorder to order in marching locusts. Science 312, 1402-1406

20 Ballerini, M. et al. (2008) Interaction ruling animal collective behavior depends on topological rather than metric distance: Evidence from a field study. Proc. Natl. Acad. Sci. U. S. A. $105,1232-1237$

21 Nagy, M. et al. (2010) Hierarchical group dynamics in pigeon flocks. Nature 464, 890-893

22 D'Eath, R.B. (1998) Can video images imitate real stimuli in animal behaviour experiments? Biol. Rev. 73, 267-292

23 Baldauf, S.A. et al. (2008) Technical restrictions of computer-manipulated visual stimuli and display units for studying animal behaviour. Ethology 114, 737-751

24 Moiseff, A. and Copeland, J. (2010) Firefly Synchrony: A behavioral strategy to minimize

434 visual clutter. Science 329, 181 
43926 Tiedemann, A. et al. (1999) Electronic (fenceless) control of livestock. Techn. Report 440 PNW-RP-510, United States Department of Agriculture, Forest Service

441

27 Butler, Z. et al. (2006) From robotics to animals: virtual fences for controlling cattle. Int. J. Robot. Res. 25, 485-508

28 Schwager, M. et al. (2008) Data-driven identification of group dynamics for motion prediction and control. J. Field Robot. 25, 305-324

29 Dyer, J.R.G. et al. (2009) Leadership, consensus decision making and collective behaviour

30 Conradt, L. et al. (2009) "Leading according to need" in self-organizing groups. Am. Nat. 173, 304-312

453

31 Sato, H. et al. (2009) Remote radio control of insect flight. Front. Integr. Neurosci. 3, 24. doi: 10.3389/neuro.07.024.2009

32 Sato, H. and Maharbiz, M.M. (2010) Recent developments in the remote radio control of insect flight. Front. Neurosci. 4, 199. doi: 10.3389/fnins.2010.00199

35 Reaney, L.T. (2009) Female preference for male phenotypic traits in a fiddler crab: do females use absolute or comparative evaluation? Anim. Behav. 77, 139-143 
47337 Ward, A.J.W. et al. (2008) Quorum decision-making facilitates information transfer in fish 474 shoals. Proc. Natl. Acad. Sci. U. S. A. 105: 6948-6953

475

38 Sumpter, D.J.T. et al. (2008) Consensus decision-making by fish. Current Biology 18: $1773-1777$

39 Krause, J. and Ruxton, G.D. (2011) Living in groups: selected topics. In: Social behaviour: 480 Genes, ecology and evolution (Szekeley T, Moore A, Komdeur J eds). Cambridge University Press, Cambridge

482

40 Couzin, I.D. et al. (2002) Collective memory and spatial sorting in animal groups. $J$.

41 Camazine, S. et al. (2001) Self-organization in biological systems, Princeton University Press

42 Sumpter, D.J.T. and Pratt, S.C. (2009) Quorum responses and consensus decision making.

43 Conradt, L. and List, C. (2009) Group decisions in humans and animals: a survey. Philos.

44 Kirchner, W.H. and Towne, W.F. (1994) The sensory basis of the honeybees dance 496 language. Sci. Am. 270, 74-80

45 King, A.J. et al. (2008) Dominance and affiliation mediate despotism in a social primate.

499 Curr. Biol. 18, 1833-1838

46 Alcock, J. (2009) Animal behaviour: an evolutionary approach. Sinauer Associates 
508

48 Krause, J. et al. (2010) Personality in the context of social networks. Philos. Trans. R. Soc. B 365: 4009-4016

49 Croft, D.P. et al. (2008) Exploring Animal Social Networks. Princeton University Press

50 Krause, J. et al. (2007) Social network theory in the behavioural sciences: potential applications. Behav. Ecol. Sociobiol. 62, 15-27

51 Flack, J.C. et al. (2006) Policing stabilizes construction of social niches in primates. Nature 439, 426-429

52 McDonald, D.B. (2007) Predicting fate from early connectivity in a social network. Proc. Natl. Acad. Sci. U. S. A 104, 10910-10914

53 Mottley, K and Giraldeau, L.A. (2000) Experimental evidence that group foragers can converge on predicted producer-scrounger equilibria. Anim. Behav. 60, 341-350

54 Grasmuck, V. and Desor, D. (2002) Behavioural differentiation of rats confronted to a complex diving-for-food situation Behav. Proc. 58, 67-77

55 Nehaniv, C. and Dautenhahn, K. (eds) (2007). Imitation and Social Learning in Robots, Humans and Animals. Cambridge University Press

56 Gribovskiy, A et al. (2010). Towards Mixed Societies of Chickens and Robots. In Proc. of the 2010 IEEE/RSJ International Conference on Intelligent Robots and Systems (IROS) 42224228.

57 Urbanek, R.P. et al. (2010) Winter release and management of reintroduced migratory Whooping Cranes Grus americana. Bird Cons. Inter. 20, 43-54

58 Couzin, I.D. (2009) Collective cognition in animal groups. Trends Cogn. Sci. 13, 36-43

(1) 
53959 Krause, J. et al. (2010) Swarm intelligence in animals and humans. Trends Ecol. Evol. 25, $540 \quad 28-34$

54260 Reebs, S.G. (2000) Can a minority of informed leaders determine the foraging movements 543 of a fish shoal? Anim. Behav. 59, 403-409

544

54561 Şahin, E. and Winfield, A.F.T. (2008) Special issue on swarm robotics. Swarm Intelligence $546 \quad 2,69-72$

54862 Floreano, D. et al. (2007) Evolutionary conditions for the emergence of communication in 549 robots. Curr. Biol. 17, 514-519

55163 Floreano, D. and Keller, L. (2010) Evolution of adaptive behaviour in robots by means of 552 Darwinian selection. PLoS Biol. 8, e1000292

553

55464 Levi, P. and Kernbach S. (eds.) (2010) Symbiotic Multi-Robot Organisms: Reliability, 555 Adaptability, Evolution. Springer

556

557

558

559 
Animal personality: individual consistency in behaviour across time and/or contexts.

563 Autonomous robot: a robot with sensory input, decision-making capabilities and behavioural 564 output.

Cognitive ability: information-processing ability in connection with problem solving.

Collective behaviour: the field of collective behaviour investigates the emergence of grouplevel properties from interactions between individuals.

Cyborg: an organism with both biological and electronic parts.

Consensus decision: agreement among group members on one course of action.

Quorum: a threshold number of individuals that, once reached, will lead to a behaviour or action for the whole group (see also consensus decision).

Robot: a machine that is able to physically interact with its environment and perform some sequence of behaviours, either autonomously or by remote control.

Self-organisation: individuals follow local behavioural rules, resulting in organised behaviour by the whole group without the need for global control.

584 Swarm intelligence: Collective behaviors, in both natural and artificial systems of multiple 585 agents, that exhibit group-level cognition.

587 Swarm robotics: the design and engineering of artificial robot swarms based on the 588 principles of swarm intelligence. 
591 Autonomous robot: a robot with sensory input that is capable of determining its next action 592 (both what action to take and when to take it) without human intervention. It is autonomous in 593 the sense that it can make and execute decisions based on its own assessment of its 594 environment. Autonomous robots are capable of interaction with live animals without human 595 guidance. An example of this type of robot is the cockroach-robot (Fig. 1) which was used to 596 investigate communal shelter selection (see section on Robots in behavioural experiments).

598 Cyborg: an organism with both biological and electronic parts; the latter allow direct control 599 of an animal by manipulating its nervous system. This control can be used for manipulating 600 the animal's locomotion or social interaction with conspecifics. The control of flight 601 performance in beetles provides an example of this novel approach to controlling animal 602 behaviour (Box 1).

603

604 Remote-controlled robot: a robot whose behaviour is controlled externally (in contrast to an 605 autonomous robot whose control-centre is inside the robot itself) by a human observer or a computer outside the robot. The robotic fish (Box 2) and the robotic bee [7] are recent examples of this kind of approach.

Smart collars: a device that can be mounted on an animal (usually in the form of a collar around the neck), which provides negative feedback if the animal enters an area where it is not supposed to go. The negative feedback consists of weak electric shocks or repellent noises and is triggered by a GPS-unit inside the collar that locates the animal's position, or an underground wire. This approach is used to retain domestic animals within certain boundaries without the use of fences (see section on Interactive technology). 


\section{Box 1. Cyborg insects}

623

624 A novel way to control animal behaviour is to directly stimulate the neural system of an 625 organism. An impressive example of such a "cyborg-approach" is the remote-control of insect 626 flight [31-33]. A radio-equipped microcontroller emits pulses via electrodes to the brain and 627 selected muscle groups. Reliable control of flight initiation, cessation, elevation and direction 628 has been possible. Two different species of beetle (a) Cotinis texana and b) Mecynorrhina 629 torquata) were used, both of which are strong enough to carry the equipment during flight.

630

\section{Costs and benefits}

632 The Cyborg-approach opens up new ways of controlling locomotion in insects that could be 633 used in many different ways to manipulate interactions between con- or heterospecifics. 634 However, some inter-individual variation in responsiveness was observed and the approach is 635 restricted to species that are strong enough to carry the equipment. Both restrictions may be 636 overcome as smaller and more sophisticated technology becomes available. There are also 637 ethical considerations to be taken into account especially if this approach were to be applied 638 to vertebrates. Furthermore, in the case of more complex social behaviours it might be 639 necessary to show that the behaviour has not become artificial in any way. For example, a 640 behavioural response might be produced that is normally not observed in a given context.

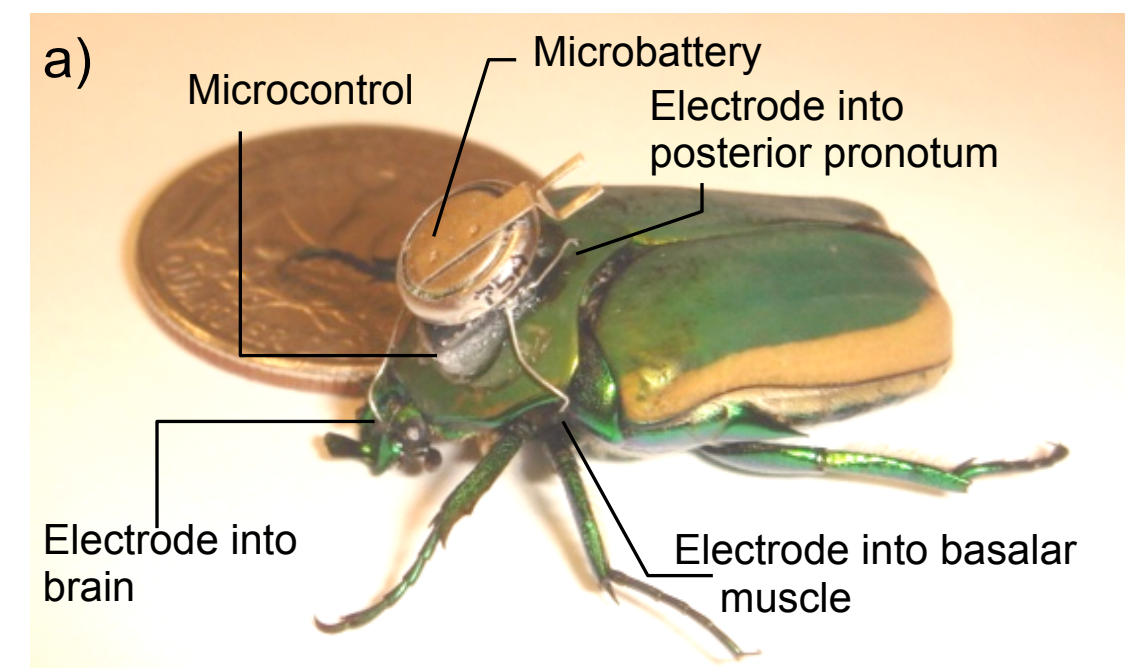




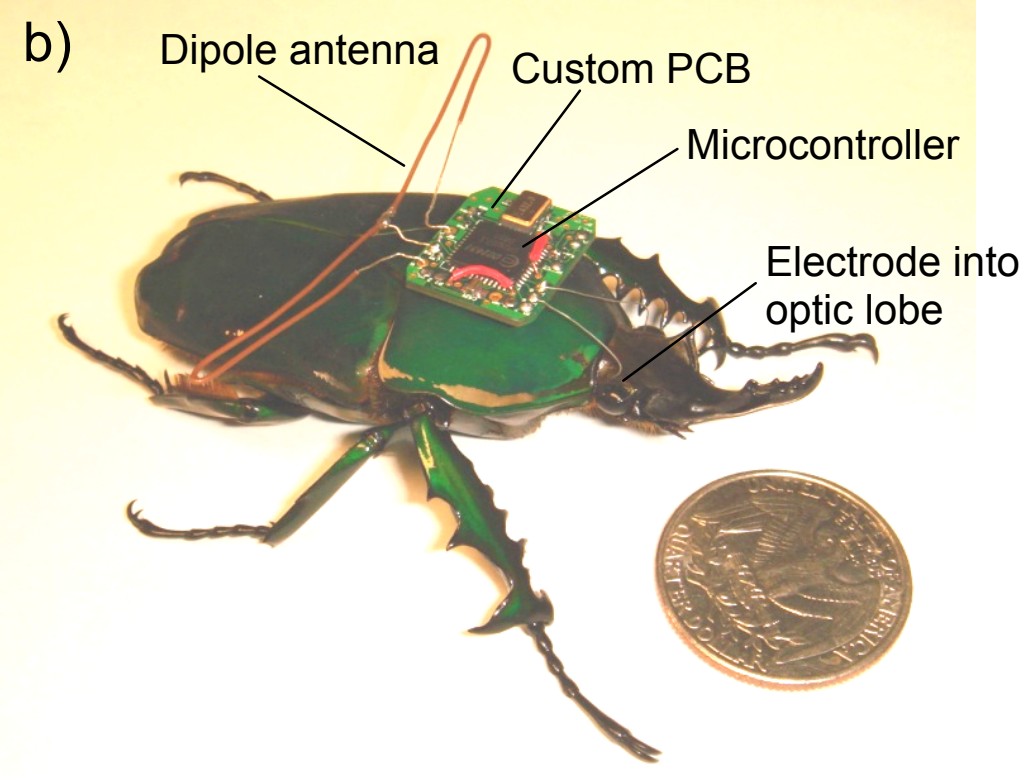

647 
650 Faria et al. [8] developed a robotic fish

651 where a dummy is mounted on a thin

652 Plexiglas rod fixed to a flat magnet and

653 guided by a robotic arm under the tank

654 that carries an electro-magnet. The

655 robotic arm is controlled via computer

656 so that the movements of the dummy

657 can be programmed. If a digital video

658 camera is positioned over the tank then

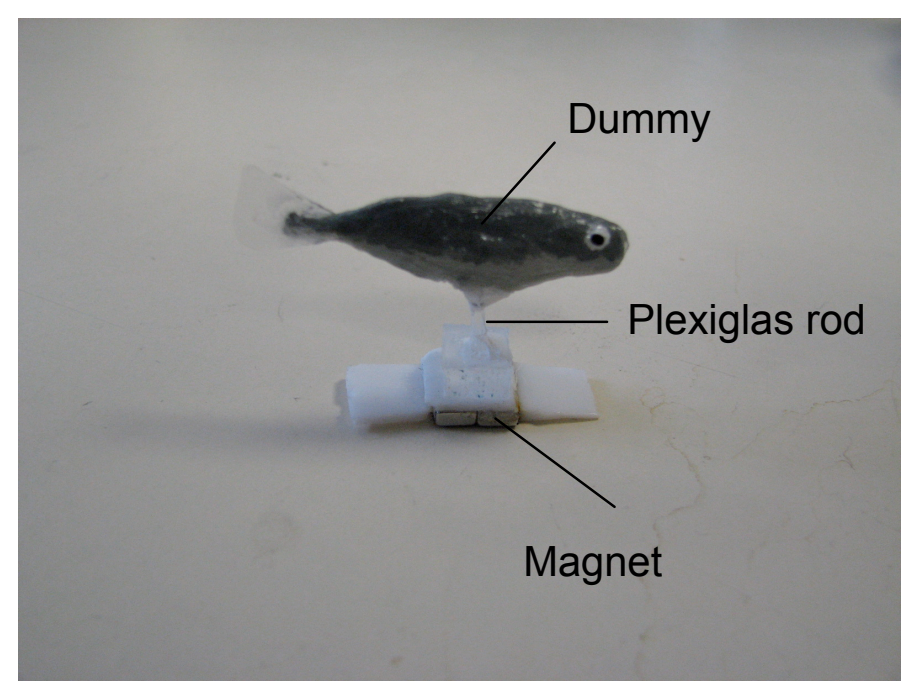

659 information on the relative position of

660 the dummy to live fish and their behaviour can be processed by a computer and behavioural

661 responses of the dummy can be initiated via the robotic arm. This would close the feedback

662 loop and allow interactions with live fish. If small remote-controlled devices are used under

663 the tank to carry electro-magnets instead of a robotic arm, then multiple dummies can be

664 controlled and moved at the same time.

665

666 Costs and benefits

667

668 The advantage of this system over autonomous robots lies in the fact that the control system is

669 separated from the dummy. This means that the same control system can now be used for all

670 kinds of dummies which can be produced in large number at low cost and quickly exchanged.

671 This approach is not limited to fish or aquatic systems but could be adopted for most

672 organisms that are small enough so that experiments can fit into an arena of a few square

673 metres. The system is relatively low cost because it only requires a standard PC, several

674 electro-motors and controllers. Potential costs are that this system can only be used in the

675 laboratory (outdoor use is, however, not necessarily straight forward with autonomous robots

676 either) and the dummies have a range that is restricted to that of the two-dimensional arena

677 which is monitored by the camera and serviced by the robotic arm.

678

679

680

681 
681 Figure 1. Interactive autonomous robot which can interact with cockroaches. It carries the 682 olfactory signature of a cockroach and is therefore treated as a conspecific by cockroaches.

683

684

685

686

687

688

689

690

691

692

693

694

695

696

697

698

699

700

701

702

703

704

705

706

707

708

709

710

711 